\title{
A Importância da Qualificação do Preceptor nos Cenários de Formação em Oncologia dos Programas de Residências em Ấrea Profissional da Saúde
}

https://doi.org/10.32635/2176-9745.RBC.2020v66n3.953

\author{
The Importance of the Preceptor's Qualification in the Oncology Training Scenarios of Residences Programs in the Professional \\ Health Area \\ La Importancia de la Calificación del Preceptor en los Escenarios de Capacitación en Oncología de los Programas de \\ Residencias en el Área de Salud Profesional
}

Laura Mata de Lima Silva'; Andressa Faria Neves Lopes²; Marina de Moraes Vasconcelos Petribú ${ }^{3}$

\section{INTRODUÇÃO}

Na última década, a educação em saúde vivenciou o aumento do número Programas de Residência na Área Profissional da Saúde, nas modalidades Multiprofissional e Uniprofissional ${ }^{1-4}$, sendo considerados um tipo de pós-graduação lato sensu , com duração mínima de dois anos, que se caracterizam pela formação por meio do trabalho, integrando ensino-serviço-comunidade, sob regime de dedicação exclusiva e supervisão docente-assistencial, de forma contextualizada e comprometida com o Sistema Único de Saúde (SUS) e suas áreas prioritárias ${ }^{3,6}$. A residência é um modelo de Educação Permanente em Saúde (EPS) ${ }^{5}$, sendo esta definida como a aprendizagem no trabalho, em que o aprender e o ensinar se incorporam ao cotidiano das organizaçóes e ao trabalho. A EPS é norteada pela Política Nacional de Educação Permanente em Saúde ${ }^{7}$, criada pela Portaria GM/MS no. 198/2004, que instituiu a necessidade de reorientação da formação profissional e de integração entre as Instituiçóes de Ensino Superior (IES), serviços de saúde e comunidade para o fortalecimento do $\mathrm{SUS}^{8}$, e normatizada atualmente pela Portaria de Consolidaçáa $\mathrm{n}^{\circ}$. 2 , de 28 de setembro de $2017^{9}$.

Residências em Área Profissional da Saúde são destinadas a todas as profissóes da saúde, excetuando-se a Medicina, a saber: Biomedicina, Ciências Biológicas, Educação Física, Enfermagem, Farmácia, Fisioterapia, Fonoaudiologia, Medicina Veterinária, Nutrição, Odontologia, Psicologia, Serviço Social e Terapia Ocupacional, incluindo, em 2014, as áreas de Física Médica e Saúde Coletiva, conforme a Portaria Interministerial MEC/MS no. 16, de 22 de dezembro de $2014^{10}$. Os Programas de Residência em Área Profissional da Saúde na modalidade Multiprofissional (RMS) devem ser constituídos por, no mínimo, três dessas profissōes. Já os Programas de Residência em Área Profissional da Saúde na modalidade Uniprofissional são destinados somente a uma profissão de saúde, conforme consta na Resolução CNRMS no. 2, de 13 de abril de $2012^{6}$. A expansão dos Programas de Residências demonstra a necessidade de especialização para o mercado de trabalho ${ }^{3}$, considerando o cenário epidemiológico, o processo de saúde-doença e as áreas prioritárias na rede de atenção à saúde do Brasil, com o objetivo de proporcionar a integralidade no cuidado ${ }^{1}$.

As neoplasias configuram-se como o principal problema de saúde pública no mundo, responsáveis pelo aumento nas taxas de morte prematura (antes dos 70 anos de idade), o que alerta para necessidade de ações de controle do câncer, nos diversos níveis de atenção (promoção da saúde, prevenção, detecção precoce, tratamento e cuidados paliativos $)^{11}$. Isso, além de outras questóes, tem estimulado a ampliaçáo de Programas de Residência com ênfase em oncologia e de vagas nos Programas já existentes. Segundo um levantamento realizado em 2015, o número de RMS em cancerologia era de aproximadamente 15 Programas, concentrados principalmente na Região Sudeste do país ${ }^{12}$. Em 2018, o relatório da Associação Brasileira de Ensino e Pesquisa em Serviço Social, sobre o mapeamento das residências em área profissional e Serviço Social, identificou 23 Programas de Residência na área de oncologia no Brasil ${ }^{13}$, demonstrando um crescimento nos

\footnotetext{
${ }^{1}$ Nutricionista. Mestre em Nutrição. Núcleo de Nutrição e Programa de Residência em Nutrição Clínica do Hospital dos Servidores do Estado. Pernambuco (PE), Brasil. Orcid iD: https://orcid.org/0000-0002-6893-9781

${ }^{2}$ Nutricionista. Especialista em Nutrição Clínica, Terapia Nutricional Enteral e Parenteral. Pós-Graduada em Nutrição e Fitoterapia, e Aprimoramento em Nutrição Oncológica. Unionco. Ambulatório de Oncologia do Grupo Rede D’OR Pernambuco e Real Instituto de Cirurgia Oncológica do Real Hospital Português de Beneficência em Pernambuco. Pernambuco (PE), Brasil. Orcid iD: https://orcid.org/0000-0002-5092-0310

${ }^{3}$ Nutricionista. Doutora em Nutrição. Docente da Universidade Federal de Pernambuco (UFPE) e Coordenadora da Comissão de Programas de Residências em Área Profissional da Saúde da UFPE. Pernambuco (PE), Brasil. Orcid iD: https://orcid.org/0000-0001-8073-0086

Endereço para correspondência: Laura Mata de Lima Silva. Rua Ilhéus, 1049, apto. 101- Piedade. Jaboatão dos Guararapes (PE), Brasil. CEP 54420-150. E-mail: laura-mata@hotmail.com
} 
últimos três anos de Programas de Residência nessa área. Ademais, nas Residências em Área Profissional da Saúde na modalidade Uniprofissional, há inserção de setores de oncologia como cenários de práticas para residentes ${ }^{14}$. A intenção é formar profissionais qualificados, pensando na interprofissionalidade e nas diretrizes para o trabalho no SUS ${ }^{3}$.

Nos Programas de Residências, vários atores contribuem para formação, como coordenadores, tutores e preceptores ${ }^{15-17}$, estando estes últimos diretamente envolvidos no processo de ensino-aprendizagem, por terem o papel desafiador de articular a prática ao conhecimento científico, dominando a área clínica de atuação e seus aspectos pedagógicos ${ }^{3,18}$. Entre as atribuiçôes do preceptor, estão: integrar o residente ao serviço e à equipe de trabalho; dar suporte e ajudar o profissional em formação a adquirir habilidades na prática clínica, até que ele fique seguro e confiante nas suas atividades ${ }^{15}$. É essencial que se estabeleça uma relação pedagógica preceptor-residente, e não somente a transferência de atividades e instrução de técnicas, reconhecendo o processo de ensino como algo inerente à prática ${ }^{3,19} \mathrm{e}$, para isso, o preceptor precisa ter planejamento, competência e criatividade. A falta de percepçáo, neste sentido, e de seu papel como educador, pode trazer lacunas para a formação do residente ${ }^{3}$.

\section{DESENVOLVIMENTO}

\section{PRECEPTOR E SUA FORMAÇÃO}

O preceptor é o profissional que orientará o residente durante a formação, utilizando seus conhecimentos e experiências, ajudando-o a se adaptar ao exercício da profissão; desenvolver competência clínica ${ }^{16}$; lidar com o ambiente de trabalho e situaçôes clínicas reais; e solucionar problemas identificados no cotidiano da atenção em saúde com suas próprias estratégias ${ }^{15}$. Ao assumir a atividade de ensino, o preceptor precisa ampliar seus conhecimentos para respaldar sua conduta, refletindo na própria formação, já que os conhecimentos relativos à profissão evoluem e há uma necessidade de formação contínua e aperfeiçoamento. A competência técnica não pode estar dissociada da competência pedagógica, mas integradas para proporcionar a construção de um conhecimento significativo $^{18}$, principalmente na oncologia, uma área que demanda açóes e serviços de saúde especializados, exigindo, portanto, profissionais com formação especializada para um cuidado de excelência ${ }^{14}$.

Acredita-se que a residência seja uma experiência importante na formação profissional e até ofereça uma base para os residentes atuarem como futuros preceptores. Entretanto, a absorção dos profissionais residentes, especializados em oncologia e capacitados para a rede de atenção do SUS, ainda náo é efetiva e continua sendo pauta de discussão nos Fóruns Nacionais de Residentes em Saúde ${ }^{12}$. $\mathrm{O}$ ensino em saúde é uma estratégia para ampliar a capacidade de enfrentamento do câncer, e uma formação adequada deve estar em consonância com mudanças demográficas e epidemiológicas, de modo que a força de trabalho detenha as competências necessárias para atender à população ${ }^{17}$.

A estrutura curricular de Programas de RMS em Oncologia, como o do Instituto Nacional de Câncer José Alencar Gomes da Silva (INCA) ${ }^{17}$ e o do A.C. Camargo Cancer Center $^{20}$, propóe a capacitação para atuação em oncologia nas diferentes fases do tratamento, o que requer um preceptor experiente na rede de atenção oncológica, e com domínio sobre os pressupostos teóricos relacionados à prática profissional, para a supervisão do residente. Isso é essencial, seja nas residências com ênfase em cancerologia, como em quaisquer Programas que incorporam os cenários de atenção ao usuário com câncer, a fim de formar profissionais qualificados e com visão humanística diferenciada para o manejo do paciente oncológico ${ }^{14}$.

O preceptor é considerado docente-clínico, mas não é um profissional da academia, e carece de formação didático-pedagógica que nem sempre é contemplada nos Programas de Residências, sendo isto um problema ${ }^{16,21,22}$. A articulação entre as instituiçóes formadoras e executoras (proponentes) dos Programas, estabelecendo parcerias entre as Universidades e as Unidades de Saúde, pode favorecer essa capacitação pedagógica dos preceptores. Como exemplo disso, a Comissão de Residência Multiprofissional (COREMU) da Universidade Federal de Pernambuco (UFPE), responsável por 11 Programas de Residência, tem se mobilizado juntamente com os coordenadores de Programas para a realização de cursos de formação didático-pedagógica voltados para a capacitação de preceptores ${ }^{23}$. Além disso, os coordenadores de Programas podem se articular com o setor de educação permanente, dentro da própria instituição, a fim de viabilizar a realização de oficinas e encontros pedagógicos in loco como parte da jornada de trabalho, com o objetivo de promover a reflexão sobre o fazer preceptoria.

Capacitaçôes que permeiam o ambiente de trabalho podem minimizar a sobrecarga de trabalho dos profissionais de saúde, a qual é um aspecto limitante para a formação pedagógica $^{22}$, juntamente com a elevada rotatividade de profissionais, o sucateamento das condiçóes de trabalho e o subfinanciamento dos Programas de Residências para a formação dos preceptores, visto que este é limitado apenas ao pagamento de bolsas aos residentes, dificultando a realização de cursos que demandam custos e a capacitação efetiva de preceptores ${ }^{5}$. Conhecer profundamente um conteúdo e obter sucesso na carreira não são condiçôes 
únicas para o exercício da preceptoria, a qual pressupóe estímulos constantes de reflexão e alternativas para conduzir o processo de ensino-aprendizagem. Alguns profissionais ainda têm uma visão hierarquizada dentro desse processo, no qual o docente é visto como "a fonte do conhecimento", não se permitindo estabelecer novos métodos de ensino-aprendizagem, integralidade e interdisciplinaridade. Geralmente, preceptores que foram residentes têm uma postura distinta ${ }^{24}$, mostrando que a formação de origem e a experiência na área na qual atuam pode ter um impacto sobre a qualidade da orientação/supervisão exercida pelos profissionais dentro dos Programas de Residências.

Pensando nessa formação do residente como futuro preceptor, seria relevante uma reflexão para revisão das diretrizes curriculares dos Programas de Residência, visando a uma formação holística de profissionais preceptores para o SUS, fazendo-se necessária a inclusão de disciplinas teórico-práticas que auxiliassem no desenvolvimento de competências pedagógicas durante o curso do Programa de Residência, qualificando assim os residentes para atuarem como preceptores, além de contribuir para a formação dos próprios profissionais da instituição, que precisariam abordar tal temática como "docentes" dos residentes.

Considerando ainda a realidade de alguns serviços, os profissionais que são preceptores não recebem remuneraçáo diferenciada e podem ter sobreposição das atividades de ensino e assistência, sem liberação de carga horária para atualização técnica e capacitação ${ }^{5}$. A ausência de políticas públicas de formação e da instituição formal do exercício da preceptoria nos Programas pode dificultar a valorização do preceptor e até desestimular o desempenho dessa atividade, prejudicando a formação e a qualidade dos Programas de Residências ${ }^{5,16}$, embora a Portaria que regulamenta a EPS preconize que esta deve ter seu espaço garantido dentro da carga horária dos trabalhadores, a fim de proporcionar a qualificaçáo de todos que fazem parte da equipe multiprofissional, bem como dos gestores? ${ }^{9}$ Em contrapartida, já existem leis brasileiras municipal ${ }^{25}$ e estadual ${ }^{26}$ que instituíram a gratificação e regulamentaram a atividade de preceptoria nas residências em área profissional da saúde nas modalidades Multi e Uniprofissionais, fortalecendo a educação permanente e a capacitação de trabalhadores para o SUS.

\section{CONCLUSÃO}

A preceptoria requer planejamento pedagógico, autoavaliação e interdisciplinaridade do profissional educador. Percebe-se que o preceptor ideal deve ter habilidades técnicas e pedagógicas, e que estas podem ser adquiridas por uma capacitação e formação específica, semelhante ao conhecimento relacionado à profissão, que envolve a academia, a especialização e a vivência prática. A formação de profissionais para rede de atenção oncológica do SUS deve ser pensada como estratégia para o controle da doença, objetivando o egresso de profissionais altamente qualificados do ponto de vista técnico-pedagógico dos Programas de Residência e com formação holística para atuarem como futuros preceptores, e que, para tal, devem ter como preceptores pessoas com expertise na área de formação na qual estão inseridas ou no respectivo cenário de prática, uma vez que o câncer é uma doença de curso clínico, geralmente lento, prolongado e permanente, demanda hospitalizaçáo e acompanhamento ambulatorial, o que requer uma equipe multiprofissional altamente especializada.

\section{CONTRIBUIÇÕES}

Todos os autores contribuíram substancialmente na concepção e/ou no planejamento do estudo; na obtenção, na análise e/ou interpretação dos dados; na redação e/ou revisão crítica; e aprovaram a versão final a ser publicada.

\section{DECLARAÇÃO DE CONFLITO DE INTERESSES}

Nada a declarar.

\section{FONTES DE FINANCIAMENTO}

Não há.

\section{REFERÊNCIAS}

1. Sarmento LF, França T, Medeiros KR, et al. The regional distribution of the provision of training in the Multi-professional Residency in Health modality. Saúde Debate. 2017;41(113):415-24. doi: https://doi. org/10.1590/0103-1104201711306

2. Silva LB. Residência multiprofissional em saúde no Brasil: alguns aspectos da trajetória histórica. Rev katálysis. 2018;21(1):200-9. doi: https://doi.org/10.1590/1982$02592018 \mathrm{v} 21 \mathrm{n} 1 \mathrm{p} 200$

3. Torres RBS, Barreto ICHC, Freitas RWJF, et al. Estado da arte das residências integradas, multiprofissionais e em área profissional da Saúde. Interface (Botucatu). 2019;23:e170691. doi: https://doi.org/10.1590/ Interface. 170691

4. Brasil. Ministério da Educação. Comissão Nacional de Residência Multiprofissional em Saúde. Resolução CNRMS no 1, de 27 de dezembro de 2017. Dispóe sobre o número de Programas de Residência em Área Profissional da Saúde, nas modalidades multiprofissional 
e uniprofissional, cursados por egressos de Programas. Diário Oficial da União, Brasília, DF; 2017 dez 29. Seção I, p. 31.

5. Silva LS, Natal S. Residência multiprofissional em saúde: análise da implantação de dois programas pela Universidade Federal de Santa Catarina, Brasil. Trab Educ Saúde. 2019;17(3):e0022050. doi: https://doi. org/10.1590/1981-7746-sol00220

6. Brasil. Ministério da Educação. Comissão Nacional de Residência Multiprofissional em Saúde. Resolução CNRMS no 2, de 13 de abril de 2012. Dispóe sobre Diretrizes Gerais para os Programas de Residência Multiprofissional e em Área Profissional de Saúde. Diário Oficial da União, Brasília, DF; 2012 abr 16. Seção I, p. 24.

7. Almeida JRS, Bizerril DO, Saldanha KGH, et al. Educação permanente em saúde: uma estratégia para refletir sobre o processo de trabalho. Rev ABENO. 2016;16(2):7-15.

8. Brasil. Ministério da Saúde. Portaria no 198, de 13 de fevereiro de 2004. Institui a Política Nacional de Educação Permanente em Saúde como estratégia do Sistema Único de Saúde para a formação e o desenvolvimento de trabalhadores para o setor e dá outras providências. Diário Oficial da União, Brasília, DF; 2004 fev 16. Seção I, p. 37.

9. Brasil. Ministério da Saúde. Portaria de Consolidação no 2 , de 28 de setembro de 2017. Consolidação das normas sobre as políticas nacionais de saúde do Sistema Único de Saúde. Diário Oficial da União, Brasília, DF; 2018 abr 13. Seção I, p. 76.

10. Brasil. Ministério da Educação. Portaria Interministerial no 16 , de 22 de dezembro de 2014. Altera a Portaria Interministerial $n^{\circ}$ 1.077/MEC/MS, de 12 de novembro de 2009, a Portaria Interministerial no $1.320 / \mathrm{MEC} /$ MS, de 11 de novembro de 2010 e revoga a Portaria Interministerial $\mathrm{n}^{\circ}$ 1.224/MEC/MS, de 3 de outubro de 2012, para atualizar o processo de designação dos membros da Comissão Nacional de Residência Multiprofissional em Saúde (CNRMS) e para incluir áreas profissionais para a realização de Programas de Residência Multiprofissional e em Área Profissional da Saúde. Diário Oficial da União, Brasília, DF; 2014 dez 23. Seção I, p. 21.

11. Instituto Nacional de Câncer José Alencar Gomes da Silva. Estimativa 2020: incidência de câncer no Brasil. Rio de Janeiro: INCA; 2019.

12. Torres RBS, Barreto ICHC, Carvalho JB. Conflitos e avanços na implementaçáo de uma Residência Integrada em Saúde com ênfase em Cancerologia. ABCS Health Sci. 2015;40(3):370-6. doi: https://doi.org/10.7322/ abcshs.v40i3.824

13. Grupo de Estudos e Pesquisas dos Fundamentos do Serviço Social (GEPEFSS), Faculdade de Serviço
Social, Universidade Federal de Juiz de Fora. Relatório da pesquisa: mapeamento das residências em área profissional e serviço social. Juiz de Fora, MG: ABEPSS; 2018 [acesso 2020 mar 29]. Disponível em: http://www. abepss.org.br/arquivos/anexos/relatorio-abepss-residenc ia-201812031150396627330.pdf

14. Santana CJM, Lopes GT. O cuidado especializado do egresso da residência em enfermagem do Instituto Nacional de Câncer - INCA. Esc Anna Nery. 2007;11(3):417-22. doi: https://doi.org/10.1590/ S1414-81452007000300004

15. Botti SHO, Rego S. Preceptor, supervisor, tutor e mentor: quais são seus papéis? Rev Bras Educ Med. 2008;32(3):363-73. doi: https://doi.org/10.1590/ S0100-55022008000300011

16. Cavalcanti IL, Sant' Ana JMB. A preceptoria em um programa de residência multiprofissional em oncologia: carências e dificuldades. Rev G\&S. 2014;5(3):1045-54

17. Instituto Nacional de Câncer José Alencar Gomes da Silva. Programas de residência multiprofissional em oncologia e residência em física médica: plano de curso. 4. ed. rev. atual. Rio de Janeiro: INCA; 2019.

18. Souza SV, Ferreira BJ. Preceptoria: perspectivas e desafios na residência multiprofissional em saúde. ABCS Health Sci. 2019;44(1):15-21. doi:_https://doi.org/10.7322/ abcshs.v44i1.1074

19. Silva AS, Magalhães CR, Carvalho GP, et al. Visão de preceptores sobre programa de residência multiprofissional com ênfase em onco-hematologia em hospital oncológico referência no sul do Brasil. Res Soc Dev. 2018;7(7):e777300. doi: https://doi.org/10.17648/ rsd-v7i7.300

20. AC Camargo Cancer Center [Internet]. São Paulo: Fundação Antônio Prudente; c2019. Residência Multiprofissional [acesso 2020 mar 29]. Disponível em: https://www.accamargo.org.br/residentes-alunos/ residencia-multiprofissional

21. Pereira CSF, Tavares CMM. Significado da modalidade de preceptoria no âmbito da residência multiprofissional em saúde num Hospital Universitário. Rev Cubana Enferm. 2016;32(4):1-11.

22. Melo MC, Queluci GC, Gouvêa MV. Preceptoria de enfermagem na residência multiprofissional em oncologia: um estudo descritivo. Braz J Nurs. 2014;13(4):1-6. doi: https://doi.org/10.5935/1676-4285.20144567

23. Santos JS, Felipe DA, organizadores. Experiências em educação permanente em saúde no estado de Pernambuco: formação que se constrói em rede. Recife: Secretaria de Saúde; 2019. Seção Temática II, Cavalcanti ATA, Nunes JRV, Petribú MMV, et al. Curso de formação didático-pedagógica para preceptores: uma estratégia da educação permanente como instrumento de mudança para os programas de residências; p. 245-53. 
24. Autonomo FROM, Hortale VA, Santos GB, et al. A preceptoria na formação médica e multiprofissional com ênfase na atenção primária - Análise das publicaçóes brasileiras. Rev Bras Educ Med. 2015;39(2):316-27. doi: https://doi.org/10.1590/1981$52712015 \mathrm{v} 39 \mathrm{n} 2 \mathrm{e} 02602014$

25. São Paulo (SP). Prefeitura Municipal de Mauá. Lei no 5.079 de 10 de setembro de 2015. Dispóe sobre a instituição da Rede Escola de Saúde de Mauá RESM, e dá outras providências. Diário Oficial do Município de Mauá, São Paulo, SP; 2015 [acesso 2020 mar 17]. Disponível em:_http://dom.maua.sp.gov.br/ LegislacaoNumero.aspx?LegislaID=5079\&TipoLegisl aID $=0$

26. Distrito Federal (BR). Câmara Legislativa. Lei no 6.455, de 26 de dezembro de 2019. Dispóe sobre as atividades de preceptoria nas carreiras Médica, Cirurgiāo Dentista, Enfermeiro e Assistência Pública à Saúde e dá outras providências. Diário Oficial do Distrito Federal, Brasília, DF; 2019 dez 27. Seçáo I, p. 11 [acesso 2020 mar 17]. Disponível em: http://www.sinj.df.gov.br/sinj/Diario/ d727ea82-f45d-38c6-aa22-b6254ac08040/DODF\%20 246\%2027-12-2019\%20INTEGRA.pdf 